\title{
The economic evaluation of a Participatory Ergonomics programme to prevent low back and neck pain
}

\author{
Maurice Driessen $^{\mathrm{a}, \mathrm{b}}$, Judith Bosmans ${ }^{\mathrm{c}}$, Karin Proper ${ }^{\mathrm{a}, \mathrm{b}}$, Johannes Anema ${ }^{\mathrm{a}, \mathrm{b}}$, Paulien Bongers ${ }^{\mathrm{a}, \mathrm{b}, \mathrm{d}}$, \\ Allard van der Beek ${ }^{\mathrm{a}, \mathrm{b},{ }^{*}}$ \\ ${ }^{a}$ Body@Work TNO VUmc, Research Center Physical Activity, Work and Health, Amsterdam, The Netherlands \\ ${ }^{b}$ Department of Public and Occupational Health, EMGO Institute for Health and Care Research, VU University \\ Medical Center, Van der Boechorststraat 7, NL-1081 BT, Amsterdam, The Netherlands \\ ${ }^{c}$ Department of Health Sciences, EMGO ${ }^{+}$Institute for Health and Care Research, VU University, Amsterdam, \\ The Netherlands \\ ${ }^{d}$ TNO Quality of Life, Hoofddorp, The Netherlands
}

\begin{abstract}
This study was an economic evaluation conducted alongside a cluster randomised controlled trial with a follow-up of 12 months. The aim was to evaluate the cost-effectiveness and cost-benefit of the Stay@Work Participatory Ergonomics programme (PE) compared to a control group (no PE). In total, 37 departments ( $n=3047$ workers) were randomised into either the intervention (PE) or control group (no PE). During a meeting, working groups followed the steps of PE, and composed and prioritised ergonomic measures aimed to prevent low back pain (LBP) and neck pain (NP). Working groups had to implement the ergonomic measures within three months in their department. Cost data included those directly related to LBP and NP. Cost-effectiveness analyses (CEA) and cost-benefit analyses (CBA) were performed. After 12 months, health care costs and costs of productivity losses were higher in the intervention group than in the control group (the mean total cost difference was $€ 127 ; 95 \%$ CI $€-164-€ 418$ ). From a societal perspective, the CEA showed that PE was not cost-effective compared to control for LBP and NP prevalence, work performance, and sick leave. The CBA from a company perspective showed a monetary loss of $€ 78$ per worker. The PE programme was neither cost-effective nor cost-beneficial on any of the effect measures.
\end{abstract}

Keywords: economic evaluation, cost-effectiveness, cost-benefit, musculoskeletal pain, participatory ergonomics.

\section{Introduction}

The prevention of low back pain (LBP) and neck pain (NP) and their unfavourable consequences has become an important goal for workers, employers, policy makers, and health care providers.

To prevent LBP and NP, a variety of preventive strategies is available, for example: lumbar supports, advice/education on working postures and methods, physical exercise programmes, lifting aids, new chairs, and pause software programmes. Systematic literature reviews showed that, with the exception of physical exercise programmes, none of the strategies was effective to prevent LBP and NP $[1,4,8,19]$.
Another approach to prevent musculoskeletal disorders (MSD) may be participatory ergonomics (PE). Supported by the management, the implementation strategy empowers workers to change their own worksite. A systematic review found some evidence that PE can reduce MSD [23]. These results were not confirmed by three large randomised controlled trials (RCTs) concluding that PE was not effective in the prevention of MSD [7,12,20]. However, irrespective of the effectiveness of an intervention, insight into its costs is important in order to decide whether or not to undertake it. So far, very little is known about the cost-effectiveness and the cost-benefit of PE when used as a strategy to prevent LBP and NP [25].

\footnotetext{
* Corresponding and presenting author. E-mail: a.vanderbeek@vumc.nl.
} 
Therefore, the present study reports on the results of an economic evaluation performed alongside a cluster RCT comparing an intervention group (PE) with a control group (no PE).

\section{Materials and Methods}

An economic evaluation from the societal and the employer's perspective was conducted alongside a cluster RCT. The RCT was conducted at four Dutch companies: a railway transportation company, an airline company, a university including its university medical hospital, and a steel company. More details on the study design and methods have been described elsewhere [5].

\subsection{Study population}

Randomisation took place at the department level, and was performed by an independent research assistant using a computer-generated randomisation programme. Departments were randomly allocated to either the intervention group (PE) or the control group (no PE). All workers within the participating departments were allowed to participate in the study. Workers meeting the following criteria at baseline were included in the analyses: 1) aged between 18-65 years; 2) not pregnant; and 3) no cumulative sick leave period longer than four weeks due to LBP or NP in the past three months.

\subsection{Control and intervention}

Before filling out the baseline questionnaire workers from both intervention and control departments were requested to watch three short (45 seconds) educative movies about the prevention of LBP and NP. The movies were used as a sham intervention and can be considered as an ineffective strategy to prevent LBP and NP [1].

Intervention departments received the Stay@Work PE programme, which has been described in detail elsewhere [5]. Briefly, under the guidance of a trained ergonomist a working group (consisting of both workers and management) followed the steps of the PE programme during a six-hour working group meeting. By following these steps, the working group brainstormed about, evaluated, and prioritised a top three risk factors for LBP and NP. Subsequently, the working group brainstormed about, evaluated, and prioritised a top three of ergonomic measures. All information about the prioritised risk factors and ergonomic measures were written down in an implementation plan. The working group had to implement the prioritised ergonomic measures within three months at their department. To enhance chances of implementation of the measures, some working group members from each working group followed a four-hour implementation training [5].

All together working groups prioritised 66 ergonomic measures: 32 individual ergonomic measures (i.e. improving awareness regarding ergonomics, worksite visits, and physical activity programmes), 27 physical ergonomic measures (i.e. ergonomic redesign or modification, new equipment, and manual handling aids), and seven organisational ergonomic measures (i.e. pause software installation, job rotation, and restructuring management style). Approximately one third $(34 \%)$ of the prioritised ergonomic measures were really implemented in the intervention departments [6].

\subsection{Data collection}

Measures of effect were the prevalence of LBP and NP, work performance, and all cause sick leave from work in days. Effect measures were assessed at baseline, three-, six-, nine-, and 12-months follow-up. Data on self-reported LBP and NP was collected using the Dutch Musculoskeletal Questionnaire (DMQ) [13]. The DMQ separately asked about the presence of LBP in the past three months and the presence of NP in the past three months: "no, never", "yes, sometimes", "yes, regularly", "yes, always". For the analyses of we dichotomised these variables by combining "no, never" and "yes, sometimes" into "no LBP or NP", and combining, "yes, regularly" and "yes, always" into "yes, LBP or NP".

Self-reported work performance was measured using a single-item question from the WHO Health Productivity Questionnaire asking workers to report their overall work performance in the past three months on an 11-point rating scale, ranging from 0 "worst performance" to 10 "top performance" $[14,15]$.

Sick leave was measured using a single item question asking workers about full days of absence from work due to all cause sick leave in the past three months [3].

Health care costs included the costs of the visits to health care providers, diagnostic examinations, and both prescribed and over-the-counter medication due to LBP and NP. Costs were expressed in 2008 Euros. 
Standard costs from the Dutch Manual for Costing were used to value the health care utilisation [21].

Costs of prescribed and over-the-counter medication were obtained from the individual worker. Health care costs were measured using questionnaires with a recall period of three months at baseline and after three-, six-, nine-, and 12-months follow-up. Participants who reported to have no LBP and/or NP complaints in the past three months were considered to have generated no LBP and/or NP related health care costs. Intervention costs encompassed the costs that were related to the development and conduct of the PE programme, and were determined via a bottom-up calculation. Costs included: study protocol development, ergonomists training costs, and ergonomists costs for guiding the working groups. Costs of the total (work) time invested by the 98 working group members to prepare and to attend the six-hour working group meeting were calculated by multiplying the total amount of hours by an average hourly wage rate $[6,21]$. Also incorporated in the intervention costs were the costs of the four-hour implementation training and encompassed: protocol development, ergonomists costs for guiding the training, and costs for the 40 workers who attended the training. Furthermore, costs for room rental, refreshments and training materials (i.e. laptop and beamer) were included [21].

Productivity loss from paid work was quantified in terms of net cumulative number of work days on all cause sick leave over a period of 12 months. The cumulative number of work days of sick leave was converted into work-hour equivalents based on a Dutch average of 1540 work hours per year [21]. Using the Friction Cost Approach (FCA), costs of production losses were calculated by multiplying the number of sick leave hours by the estimated price of production loss of a worker per hour of sick leave, based on age and gender. The FCA assumes that costs are limited to the friction period (i.e. the time it takes to find a replacement. A friction period of 154 calendar days and an elasticity of 0.8 were used $[16,21]$.

\subsection{Data analysis}

The economic evaluation was performed according to the intention-to-treat principle. Baseline characteristics of workers in the two groups were compared using descriptive statistics. Due to the amount of missing follow-up data, missing data were imputed using multiple imputation (MI) based on Mul- tivariate Imputation by Chained Equations (MICE) [27]. The MI procedure was performed in SPSS 17.0, in which five complete data sets were generated. By using Rubin's rules, SPSS enabled to pool effects and costs from the five complete data sets.

For the cost-effectiveness analysis (CEA), incremental cost-effectiveness ratios (ICERs) were calculated by dividing the differences between groups in costs by those in effects. For the outcomes LBP and NP prevalence and work performance, total societal costs (health care, intervention and lost productivity costs) were used. For the outcome all cause sick leave total health care costs and intervention costs were concerned. The ICER indicates the additional investments needed for the intervention to gain one extra unit of effect compared to the control group.

In the cost-benefit-analysis (CBA), a net company benefit was calculated by subtracting the difference in intervention costs between the two groups from the difference in lost productivity costs between the groups (benefits).

The 95\% CI around the mean cost differences and the uncertainty around the ICERs were estimated using bootstrapping with 5000 replications [2,9]. To graphically illustrate the uncertainty around the ICERs, we plotted each of the bootstrapped costeffect pairs in a cost-effectiveness plane. All costs, effects, cost-effectiveness and cost-benefit analyses were based on imputed data. MI and data processing was performed in SPSS 17.0, and all analyses were performed in $\mathrm{R}$.

\section{Results}

The randomisation procedure allocated 19 departments to the intervention group and 18 departments to the control group. All included 3047 workers ( $\mathrm{n}=$ 1472 intervention group, and $n=1575$ control group) were approached for the follow-up measurements. No meaningful differences between the two groups were found at baseline. Loss to follow-up on the primary outcome measure (the prevalence of LBP and NP) was considerable. After six months, 511 workers $(35 \%)$ in the intervention group and 464 workers $(29 \%)$ in the control group did not fill out their questionnaires. After 12 months, 594 workers (40\%) in the intervention group and 580 workers $(37 \%)$ in the control group did not respond on the questionnaire. Complete follow-up data was derived from 1280 workers on the two primary outcome measures (LBP and NP) and from 111 workers $(8.6 \%)$ on the health 
care costs. At baseline, workers with and workers without complete follow-up did not differ on both primary outcome measures.

\subsection{Costs and effects}

The costs of PE were $€ 29$ per intervention group worker. Total health care costs and productivity loss costs were higher in the intervention group compared to those in the control group, but the cost differences were not statistically significant. Mean total costs in the intervention group were $€ 127$ higher than those in the control group, but this difference was also not statistically significant $(95 \%$ CI $-164-418)$.

No statistically significant differences were found between the intervention group and the control group on LBP prevalence $(0.53 \% ; 95 \%$ CI $-3.13-4.19)$ and NP prevalence $(0.26 \% ; 95 \%$ CI $-2.71-3.23)$ at 12 months. Also, regarding the effect measures work performance ( 0.00 points; $95 \% \mathrm{CI}-0.15-0.16)$ and all cause sick leave $(0.48$ days; $95 \%$ CI $-1.45-2.42)$ no statistically significant differences between the intervention and the control group were found.

\subsection{Cost-effectiveness and cost-benefit analysis}

For LBP prevalence an ICER of 23,749 was found, meaning that $\mathrm{PE}$ was more expensive than the control group to obtain an extra unit of adverse effect. ICERs in similar directions were found for all of the other outcomes. These results indicate that for all outcomes the control group (no PE) dominated the intervention.

The CBA from a company perspective showed that the costs of $\mathrm{PE}$ were $€ 29$ per worker, while the PE programme did not result in monetary benefits (costs per worker were €49; 95\% CI -306 - 327). This indicates that PE was not cost-beneficial.

\section{Discussion}

This study evaluated the cost-effectiveness and cost-benefit of the Stay@Work PE programme (PE) versus a control group (no PE) to prevent LBP and NP among workers. No significant differences in effects on health care and lost productivity costs were found. This study found that the PE intervention evaluated was neither cost-effective nor costbeneficial compared to the control group.

\subsection{Strengths and limitations}

An important strength of the present study is its pragmatic cluster RCT design, which enabled us to study the (cost-) effectiveness and cost-benefit of PE under real world conditions. High number of workers recruited from various working settings participated in the study, which improves the external validity of our study results. Furthermore, this is the first RCT on LBP and NP prevention that has investigated the cost-effectiveness and the cost-benefit of PE.

This study has also some methodological limitations. The first limitation is the low implementation rate. All together, the working groups implemented only $34 \%$ of the prioritised ergonomic measures $(n=66)$ in the intervention departments. Probably, implementing one third of the ergonomic measures is insufficient to obtain an effect on LBP and NP prevention. A second limitation concerns the considerable loss to follow-up on the primary outcome measures (LBP and NP). Drop-out rates after 12 months exceeded the maximum of $30 \%$ [10]. Complete follow-up on the primary outcome measures was derived from 1280 workers. However, complete (health care cost) data was available for only 111 workers resulting in no complete cases analysis. Although we imputed missing values using the MICE procedure, the missing data may have affected the internal validity of the study. We checked our data for selective drop-out. Non-responders did not differ from responders on several important prognostic LBP and NP factors (i.e. age, gender, prevalence, pain intensity and duration). We believe that the considerable number of missing values was mainly due to the preventive character of this study [11]. At baseline a substantial group of workers $(>70 \%)$ did not have LBP and NP, did not consume health care, and thereby probably did not feel the need to fill out the health care costs questionnaire.

Self-reported sick leave rates may be underestimated, and the use of company databases may have led to more valid information on this outcome. Furthermore, it is debatable whether a broad concept such as work performance can be captured by one single question [22]. Finally, cost data were collected retrospectively every three months, which may introduce recall bias. However, we do not believe these aspects have influenced our findings because similar procedures were used in both the intervention and the control group.

It is questionable whether the costs of the prioritised ergonomic measures have to be considered as 
intervention costs. We did not incorporate these costs because we purposed to evaluate the costeffectiveness and cost-benefit of PE when used as an implementation strategy. Logically, the adding up of these costs would have led to larger cost differences between the two groups.

\subsection{Comparison with the literature}

The recent systematic review by Tompa et al. (2010) reported that PE may result in financial returns when implemented as a strategy to prevent MSD. Contradictory, most RCTs do not support the use of PE to prevent MSD such as LBP and NP $[7,12,20]$. Comparing our results on the costs of PE with those of other RCTs is hampered since these studies did not conduct an economic evaluation $[12,20]$. Tsutsumi et al. (2009) showed in a RCT that PE among Japanese workers resulted in a small but statistically significant increase on job performance [26]. However, no information on the costeffectiveness or cost-benefit was available. In contrast, economic evaluations have been conducted alongside RCTs that used PE as a return to work intervention for workers who were absent from work due to LBP. One Canadian study found a trend (not statistically significant) towards cost-benefit and cost-effectiveness in favour of PE [18]. In the Dutch setting, PE proved to be cost-effective compared to usual care $[17,24]$. The results of Steenstra et al. (2006) showed that an additional $€ 19$ resulted in a sick leave reduction of one day [24]. Lambeek et al. (2010) found that the extra investment of $€ 4$ in an integrated care programme (including PE) resulted in one day earlier return to work among workers with chronic LBP [17]. Moreover, the CBA revealed that every euro invested in intervention programme would lead to a return on investment of $€ 26$ [17].

\subsection{Implications and conclusion}

Economic evaluations are important for decision makers in health care and for managers in companies, even when interventions are not effective. Evidence on the cost-effectiveness and cost-benefit is important to decide whether or not to undertake an intervention. Researchers, and especially those who conduct RCTs, are recommended to report on the costeffectiveness and cost-benefit of PE when used to prevent MSD (i.e. LBP and NP). To improve the success of PE, researchers should take into account, preferably in advance of conducting a PE programme, the difficulties accompanied with the implementation of ergonomic measures.

In conclusion, the PE programme evaluated in this study was neither cost-effective nor cost-beneficial in preventing LBP and NP.

\section{References}

[1] Bigos SJ, Holland J, Holland C et al. High-quality controlled trials on preventing episodes of back problems: systematic literature review in working-age adults. Spine J 2009;9:147-68.

[2] Briggs AH, Wonderling DE, Mooney CZ. Pulling costeffectiveness analysis up by its bootstraps: a non-parametric approach to confidence interval estimation. Health Econ 1997;6:327-40.

[3] Burdorf A, Post W, Bruggeling T. Reliability of a questionnaire on sickness absence with specific attention to absence due to back pain and respiratory complaints. Occup Environ Med 1996;53:58-62.

[4] Burton AK, Balague F, Cardon G et al. Chapter 2. European guidelines for prevention in low back pain: November 2004. Eur Spine J 2006;15 Suppl 2:S136-S168.

[5] Driessen MT, Anema JR, Proper KI et al. Stay@Work: Participatory Ergonomics to prevent low back and neck pain among workers: design of a randomised controlled trial to evaluate the (cost-)effectiveness. BMC Musculoskelet Disord 2008;9:145.

[6] Driessen MT, Proper KI, Anema JR et al. Process evaluation of a Participatory Ergonomics programme to prevent low back pain and neck pain among workers. Implement Sci 2010;5:65.

[7] Driessen MT, Proper KI, Anema JR et al. The effectiveness of Participatory Ergonomics to prevent low-back and neck pain results of a cluster randomized controlled trial. Scand J Work Environ Health 2011;37:383-93.

[8] Driessen MT, Proper KI, van Tulder MW et al. The effectiveness of physical and organisational ergonomic interventions on low back pain and neck pain: a systematic review. Occup Environ Med 2010;67:277-85.

[9] Flynn TN and Peters TJ. Use of the bootstrap in analysing cost data from cluster randomised trials: some simulation results. BMC Health Serv Res 2004;4:33.

[10]Furlan AD, Pennick V, Bombardier C et al. 2009 updated method guidelines for systematic reviews in the Cochrane Back Review Group. Spine 2009;34:1929-41.

[11] Galea S and Tracy M. Participation rates in epidemiologic studies. Ann Epidemiol 2007;17:643-53.

[12]Haukka E, Leino-Arjas P, Viikari-Juntura E et al. A randomised controlled trial on whether a participatory ergonomics intervention could prevent musculoskeletal disorders. Occup Environ Med 2008;65:849-956.

[13]Hildebrandt VH, Bongers PM, van Dijk FJ et al. Dutch Musculoskeletal Questionnaire: description and basic qualities. Ergonomics 2001;44:1038-55.

[14]Kessler RC, Ames M, Hymel PA et al. Using the World Health Organization Health and Work Performance Questionnaire (HPQ) to evaluate the indirect workplace costs of illness. J Occup Environ Med 2004;46:S23-S37.

[15] Kessler RC, Barber C, Beck A et al. The World Health Organization Health and Work Performance Questionnaire (HPQ). J Occup Environ Med 2003;45:156-74. 
[16]Koopmanschap MA and Rutten FF. A practical guide for calculating indirect costs of disease. Pharmacoeconomics 1996;10:460-6.

[17] Lambeek LC, Bosmans JE, van Royen BJ et al. Integrated care for sick-listed patients due to chronic low back pain leads to a high return on investment; results of an economic evaluation. BMJ 2010;30:c6414.

[18]Loisel P, Lemaire J, Poitras S et al. Cost-benefit and costeffectiveness analysis of a disability prevention model for back pain management: a six year follow up study. Occup Environ Med 2002;59:807-15.

[19] Martimo KP, Verbeek J, Karppinen J et al. Effect of training and lifting equipment for preventing back pain in lifting and handling: systematic review. BMJ 2008;336:429-31.

[20] Morken T, Moen B, Riise T et al. Effects of a training program to improve musculoskeletal health among industrial workers - Effects of supervisors role in the intervention. Int $\mathrm{J}$ Ind Ergon 2002;30:115-27.

[21] Oostenbrink, JB, Bouwmans, CAM, Koopmanschap MA et al Dutch manual for costing: methods and standard costs for economic evaluations in health care. Diemen: Health Insurance Council (CVZ), 2004.
[22] Prasad M, Wahlqvist P, Shikiar R et al. A review of selfreport instruments measuring health-related work productivity: a patient-reported outcomes perspective. Pharmacoeconomics 2004;22:225-44.

[23] Rivilis I, Van Eerd D, Cullen K et al. Effectiveness of participatory ergonomic interventions on health outcomes: a systematic review. Appl Ergon 2008;39:342-58.

[24] Steenstra IA, Anema JR, van Tulder MW et al. Economic evaluation of a multi-stage return to work program for workers on sick-leave due to low back pain. J Occup Rehabil 2006;16:557-78.

[25] Tompa E, Dolinschi R, de Oliveira C et al. A systematic review of workplace ergonomic interventions with economic analyses. J Occup Rehabil 2010;20:220-34.

[26] Tsutsumi A, Nagami M, Yoshikawa T et al. Participatory intervention for workplace improvements on mental health and job performance among blue-collar workers: a cluster randomized controlled trial. J Occup Environ Med 2009;51:554-63.

[27] van Buuren S. Multiple imputation of discrete and continuous data by fully conditional specification. Stat Methods Med Res 2007;16:219-42. 